Elsevier required licence: (C2016. This manuscript version is made available under the CC-BY-NC-ND 4.0 license http://creativecommons.org/licenses/bync-nd/4.0/ 


\title{
Trading Heterogeneity under Information Uncertainty
}

\author{
Xue-Zhong $\mathrm{He}^{1}$ and Huanhuan Zheng ${ }^{2}$ \\ ${ }^{1}$ University of Technology Sydney \\ UTS Business School \\ tony.he1@uts.edu.au \\ ${ }^{2}$ The Chinese University of Hong Kong \\ Department of Economics \\ and \\ Institute of Global Economics and Finance \\ arwenzh@gmail.com
}

May 31, 2016 


\begin{abstract}
Instead of heuristical heterogeneity assumption in the current heterogeneous agent models (HAMs), we derive the trading heterogeneity by introducing information uncertainty about the fundamental value to a HAM. Conditional on their private information about the fundamental value, agents choose different trading strategies when optimizing their expected utilities. This provides a microfoundation to heterogeneity and switching behavior of agents. We show that the HAM with trading heterogeneity originating from the incomplete information performs equally well, if not better than existing HAMs, in generating bubbles, crashes, and mean-reverting prices. The simulated time series matches with the $\mathrm{S} \& \mathrm{P} 500$ in terms of power law distribution in returns, volatility clustering and long memory in volatility.
\end{abstract}

Keywords: Information friction, heterogeneity, endogeneity, stock returns, stylized facts.

JEL: G12, D53, D83 


\section{Introduction}

Heterogeneous agent models (HAMs) are useful in explaining financial market abnormality such as bubbles and crashes (Lux, 1995; Brock and Hommes, 1998; He and Westerhoff, 2005; Huang et al., 2010). They are also powerful in duplicating and analyzing stylized facts of financial data, such as fat tails, volatility clustering and long memory (Alfarano et al., 2008; Huang et al., 2012; $\mathrm{He}$ and $\mathrm{Li}, 2015)$. More recent evidence suggests that HAMs provide empirical specifications that outperform random walk and many conventional models (de Jong et al., 2010 and Chiarella et al., 2012, Lof, 2015). The explanatory power of the existing HAMs mainly comes from exploring market mechanism by focusing more on the interaction among heterogeneous agents, but less so on the role of information friction. In particular, the HAM literature assumes a complete information about the fundamental value of risky assets. It is well recognized that information friction plays a very important role in explaining various puzzles and anomalies in financial markets. ${ }^{1}$

This paper aims to examine the joint role of heterogeneity and information uncertainty in financial markets. It contributes to the HAMs by providing an endogenous mechanism on trading heterogeneity among agents and a micro-foundation to heterogeneity and switching behavior of agents. More explicitly, we consider a HAM in which agents face information uncertainty by receiving private noisy signals about the fundamental value of a risky asset when entering the market. Due to the uncertainty about the fundamental value and well-documented price momentum in short-run, agents consider both trading strategies based on the private information and short-run momentum when making their investment decision. Conditional on public information of history price and his private signal, an agent chooses the trading strategy that maximizes his expected utility. This leads to endogenous heterogeneity and switching behavior of agents' choices. We show that the HAM with trading heterogeneity originating from the information uncertainty performs equally well, if not better than existing HAMs, in generating bubbles, crashes, and mean-reverting

\footnotetext{
${ }^{1}$ There is a growing literature that deviates from the standard fundamentalist-chartist setup. For example, Parke and Waters (2007) allow agents to utilize different subsets of the complete information, Kasa et al. (2014) introduce two information sets about the fundamental that are translated into heterogeneous beliefs.
} 
prices. Numerical analysis shows that the model is able to match with the S\&P 500 in terms of power-law distribution in returns, volatility clustering and long memory in volatility.

This paper is closely related to the current HAMs with respect to heterogeneity and switching behavior of agents. However, different from the current HAMs, the heterogeneity of agents in this paper is characterized by agents' choices between two typical trading strategies based on the long-run reversal (to the fundamentals) and short-run momentum (in price trend), the two most prominent financial market anomalies (see, for example, Poterba and Summers, 1988; Jagadeesh and Titman, 1993 and Moskowitz et al., 2012). Despite the success HAMs have achieved, many remain skeptical about this approach arguing that they rely on too many heuristic assumptions. Most of the existing HAMs either exogenously specify whether an agent is a fundamentalist or chartist (Frankel and Froot, 1990; Day and Huang, 1990) or assume that agents have a complete information about the fundamental value and switch from one type to another based on some performance measures. In the seminal work of Brock and Hommes (1998), agents switch evolutionarily to the strategy that generates higher past realized profit following a discrete choice probability function. Because of the complete information, agents are able to compare the performance of the two commonly used strategies and switch to better performed strategies. Such an approach is widely applied with different customized switching criteria in subsequent studies. ${ }^{2}$ These studies innovatively capture the behavioral aspects of trading and model how agents change their strategy over time, focusing more on the market mechanism of heterogeneous trading on market inefficiency but less (or nor) on the role of information uncertainty. Therefore they are limited to explain why not all agents from the same group switch to better-performing strategies with certainty. If the information is complete, should not all agents cluster to the strategy that is expected to perform better?

This paper aims to provide a micro-foundation on trading heterogeneity and switching behavior of agents' choices by considering information uncertainty and agents' optimal trading decision

\footnotetext{
${ }^{2}$ See for example, de Jong et al. (2010), and Jongen et al. (2012). For other switching mechanism, see Chiarella et al. (2012) that model the fraction of fundamentalists as a Markov process conditional on some unobserved market conditions, i.e. booms and burst states; Lof (2012) that updates the fractions of fundamentalists and chartists according to a discrete function of the real business cycles.
} 
facing the uncertainty. In this paper, agents are not heterogeneous by nature, experience or randomness. Instead, we assume a continuum of agents who have incomplete information about the fundamental value. Each agent receives a noisy and private signal about the fundamental value when entering the market. Because of the information uncertainty about the fundamental value, agents make their decision by considering trading strategies based on both public and private information. ${ }^{3}$ Therefore, agents are allowed to choose either fundamental trading strategy based on the private information or chartist trading strategy based on public information and price trend. Conditional on the public information and his private signal, each agent chooses the strategy that generates a higher expected utility. Due to the information dispersion, agents may choose different trading strategies, generating cross-sectional trading heterogeneity among agents. Instead of switching exogenously based on certain probability, agents switch their choices on the trading strategies endogenously based on their information and the optimal trading. As market prices and agents' private information change, the market fractions of agents choosing particular strategies vary over time. As a result, both cross-sectional and time-varying trading heterogeneity arise.

Representing enumerable strategies with fundamental and chartist trading strategies is on the one hand motivated by the survey finding (Allen and Taylor, 1990) that most investors, especially institutional investors, conduct both fundamental and technical analysis. On the other hand, this is also motivated by the long-run mean-reversal and short-run momentum in prices, the two market anomalies observed widely across various financial markets. It also follows from the conventional setup in HAMs. Even though agents are well-informed about the fundamental value, because of the information uncertainty about the fundamental value, an agent may choose chartist strategy when it is expected to generate higher expected utility conditional on public information and his private signal. In particular, when mispricing based on the private information is expected to be small, a fundamental trading strategy may lead to a lower expected utility, while a short-run momentum strategy may lead to a higher expected utility, which motivates agents to choose chartist

\footnotetext{
${ }^{3}$ With complete information assumption in the current HAMs, agents are able to compare the performance of different trading strategies. However, facing the information uncertainty, it makes agents more difficult, if not impossible, to compare the performance of these two strategies and choose the better performed one.
} 
strategy. Among various trading strategies based on the public information, we choose momentum strategy which is widely used in the HAMs and well supported by empirical evidence on short-run momentum. As a result, the fractions of agents who choose fundamental and momentum strategies are uniquely determined by the distribution of the private signals and the past asset prices.

In our model, the market fraction of agents who choose the fundamental trading strategy generally increases with the degree of asset mispricing, but declines with the market power of agents who choose momentum strategy. Here the degree of mispricing is measured by the absolute difference between market price and the expected fundamental value, while the market power is measured by the absolute difference between market price and a reference price or price trend. As the market price and private signals change, the fraction of agents who choose the fundamental trading strategy fluctuates, which affects the comparative advantage of the two strategies and hence the aggregate demand of agents. This in turn has a feedback effect on the magnitude and direction of the future price movements, generating rich price dynamic patterns. We show that the explanatory power of the model developed in this paper remains robust with information uncertainty compared to the current HAMs. In particular, the price dynamics generate occasional booms and bursts of bubbles, as well as transitions between bubbles and recessions. The simulated time series exhibit the power-law distribution in returns, volatility clustering and long memory, which are commonly observed properties in financial market returns and volatility.

\section{The Model}

We consider a continuum $[0,1]$ of agents trading on one risky asset and one risk-free asset in discrete-time. For simplicity, the interest rate of the risk-free asset is normalized to zero. The fundamental value of the risky asset $\mu$ is not known publicly. Agents only know that $\mu$ is normally distributed with mean of $\bar{\mu}$ and standard deviation of $\sigma_{\mu}$. Denote $\alpha_{\mu}=1 / \sigma_{\mu}^{2}$ the precision of the distribution of the fundamental value $\mu$. In each time period, there is a new entry of agents ${ }^{4}$ and

\footnotetext{
${ }^{4}$ Different from the existing HAMS, we allow agents to come, stay, or leave the market at any point of time.
} 
each agent $i$ receives a private signal on the fundamental value $\mu$, given by

$$
x_{i, t}=\mu+\varepsilon_{i, t},
$$

where the noise term $\varepsilon_{i, t}$ is independently and normally distributed with mean 0 and a standard deviation of $\sigma_{x}$, across agents and over time. Similarly denote $\alpha_{x}=1 / \sigma_{x}^{2}$ the precision of agents' signals. This implies that the private signals are normally distributed with a mean of $\mu$ and a variance of $1 / \alpha_{x}$. All agents have a constant absolute risk aversion (CARA) exponential utility function

$$
U\left(W_{i, t}\right)=-\exp \left(-A W_{i, t}\right)
$$

where $A$ is the common absolute risk aversion coefficient for all agents and $W_{i, t}$ is the wealth of agent $i$ in time $t$. Let $p_{t}$ be the (cum-)market price of the risky asset and denote $I_{t}=\left\{p_{t}, p_{t-1}, \cdots\right\}$ the public information of history price. Each agent seeks to maximize the expected utility by allocating her wealth between risky and risk-free assets conditional on the public information $I_{t-1}$ and her private signal $x_{i, t}$ about the fundamental value of the risky asset. Let $q_{i, t}$ be agent $i$ 's demand of the risky asset at time $t$. Then the expected utility of the agent becomes

$$
\begin{aligned}
& E\left(U\left(W_{i, t} \mid x_{i, t}, I_{t-1}\right)\right)=-\exp \left(-A\left[E\left(W_{i, t} \mid x_{i, t}, I_{t-1}\right)-\frac{A}{2} \operatorname{Var}\left(W_{i, t} \mid x_{i, t}, I_{t-1}\right)\right]\right) \\
& =-\exp \left(-A\left[W_{i, t-1}+q_{i, t}\left(E\left(p_{t} \mid x_{i, t}, I_{t-1}\right)-p_{t-1}\right)-\frac{A}{2} q_{i, t}^{2} \operatorname{Var}\left(p_{t} \mid x_{i, t}, I_{t-1}\right)\right]\right),
\end{aligned}
$$

where $E\left(p_{t} \mid x_{i, t}, I_{t-1}\right)$ and $\operatorname{Var}\left(p_{t} \mid x_{i, t}, I_{t-1}\right)$ are agent $i$ 's prediction about the price and variance of the risky asset conditional on the public information $I_{t-1}$ and her signal $x_{i, t}$. The maximization of the expected utility leads to agent $i$ 's optimal demand

$$
q_{i, t}=\frac{E\left(p_{t} \mid x_{i, t}, I_{t-1}\right)-p_{t-1}}{A \operatorname{Var}\left(p_{t} \mid x_{i, t}, I_{t-1}\right)}
$$


Note that $q_{i, t}$ is independent of the agent's wealth but relies on her predicted future price movement. It is an increasing function of the predicted price change, $E\left(p_{t} \mid x_{i, t}, I_{t-1}\right)-p_{t-1}$, and a decreasing function of the price volatility $\operatorname{Var}\left(p_{t} \mid x_{i, t}, I_{t-1}\right)$.

Facing the information uncertainty on the fundamental value, an agent considers both the longrun mean-reverting of the market price to the fundamental value and the short-run momentum in price trend when entering the market. Correspondingly, the agent considers both fundamental and momentum trading strategies based on the public information of the history price and her private signal about the fundamental value of the risky asset. More explicitly, the predicted price and variance based on fundamental trading strategy are

$$
\begin{aligned}
E^{f}\left(p_{t} \mid x_{i, t}, I_{t-1}\right) & =(1-\gamma) p_{t-1}+\gamma \frac{\alpha_{\mu} \bar{\mu}+\alpha_{x} x_{i, t}}{\alpha_{\mu}+\alpha_{x}} \\
\operatorname{Var}^{f}\left(p_{t} \mid x_{i, t}, I_{t-1}\right) & =\gamma^{2} \operatorname{Var}\left(\mu \mid x_{i, t}, I_{t-1}\right)=\frac{\gamma^{2}}{\alpha_{\mu}+\alpha_{x}},
\end{aligned}
$$

where $\gamma \in(0,1]$ is a constant. Note that $\frac{\alpha_{\mu} \bar{\mu}+\alpha_{x} x_{i, t}}{\alpha_{\mu}+\alpha_{x}}$ and $\frac{1}{\alpha_{\mu}+\alpha_{x}}$ are agent $i$ 's posterior updating of the mean and variance, respectively, of the fundamental value of the risky asset conditional on her signal $x_{i, t}$. Condition (3) means that the predicted price is a weight average of the latest market price and the posterior updating of the fundamental value conditional on her private signal $x_{i, t}$; while (4) means that the conditional variance is proportional to the posterior variance conditional on the private signal $x_{i, t}$. In particular, when $\gamma=1$, the conditional mean and variance (3)-(4) are reduced to the posterior mean and variance respectively. Condition (3) can also be written as

$$
E^{f}\left(p_{t} \mid x_{i, t}, I_{t-1}\right)-p_{t-1}=\gamma\left[\frac{\alpha_{\mu} \bar{\mu}+\alpha_{x} x_{i, t}}{\alpha_{\mu}+\alpha_{x}}-p_{t-1}\right]
$$

Therefore $\gamma$ measures the convergence rate of the market price to the expected fundamental value. A high $\gamma$ means a quick convergence to the expected fundamental value. Therefore the fundamental trading strategy reflects agent's belief that the future price is expected to converge to the expected fundamental value. Though the private signals $x_{i, t}$ are i.i.d. across agents and over time, they are 
partially incorporated through the current market prices $p_{t}$, which is then reflected in the prediction of the future price. This set up is different from the rational equilibrium model in which the expected price equals to its fundamental value in the next period. Consequently, following from (2)-(4), the optimal demand of the risky asset based on the fundamental analysis becomes

$$
q_{i, t}^{f}=\frac{\alpha_{\mu} \bar{\mu}+\alpha_{x} x_{i, t}-\left(\alpha_{\mu}+\alpha_{x}\right) p_{t-1}}{A \gamma}
$$

which is called the fundamental trading strategy $f$.

The predicted price and variance based on momentum trading are independent of the private signal $x_{i, t}$,

$$
E^{c}\left(p_{t} \mid x_{i, t}, I_{t-1}\right)=p_{t-1}+\beta\left(p_{t-1}-v_{t}\right), \quad \operatorname{Var}^{c}\left(p_{t} \mid x_{i, t}, I_{t-1}\right)=\sigma_{t-1}^{2},
$$

where $v_{t}$ is a reference price or a price trend, $\beta$ measures the extrapolation of the price deviation from the trend, and $\sigma_{t-1}^{2}$ is a heuristic prediction on the variance of the asset price. The reference price $v_{t}$ can be a moving average, a supporting (resistance) price level, or any index derived from technical analysis. Equations (2) and (6) then lead to the optimal demand of the risky asset

$$
q_{i, t}^{c}=\frac{\beta\left(p_{t-1}-v_{t}\right)}{A \sigma_{t-1}^{2}},
$$

which is called momentum strategy $c$. In particular, when $v_{t}$ is a moving average of history price and $\beta>(<) 0$, the strategy $c$ is essentially a time-series momentum (contrarian) strategy (Moskowitz et al., 2012). For now, we keep the specification of $v_{t}$ open in order to keep the model general.

Given the information uncertainty, the agent compares the expected value functions based on the two optimal trading strategies and chooses the one with relative higher value function. More explicitly, the agent firstly calculates the respective value functions based on strategy $f$ and $c$, 
which can be obtained by substituting Eqs.(5) and 7) into Eq.(1)

$$
\begin{aligned}
& E_{i, t}^{f}(U)=-\exp \left\{-A\left[W_{i, t-1}+\frac{\left[\alpha_{\mu} \bar{\mu}+\alpha_{x} x_{i, t}-\left(\alpha_{\mu}+\alpha_{x}\right) p_{t-1}\right]^{2}}{2 A\left(\alpha_{\mu}+\alpha_{x}\right)}\right]\right\} \\
& E_{i, t}^{c}(U)=-\exp \left\{-A\left[W_{i, t-1}+\frac{\beta^{2}\left(p_{t-1}-v_{t}\right)^{2}}{2 A \sigma_{t-1}^{2}}\right]\right\}
\end{aligned}
$$

The agent then compares the value functions of the two strategies and selects the one that yields a higher value function. Note that $E_{i, t}^{f}$ is an increasing function of the absolute value of the signal $\left|x_{i, t}\right|$, while $E_{i}^{c}$ is independent of $x_{i, t}$. Therefore there exists threshold values for the private signal such that $E_{i, t}^{f}=E_{i, t}^{c}$. Let $\bar{x}_{t}$ be the threshold signal value that makes agent $i$ indifferent between choosing strategies $f$ and $c$ when $x_{i, t}=\bar{x}_{t}$, that is

$$
\frac{E_{i, t}^{f}(U)}{E_{i, t}^{c}(U)}=\exp \left\{-\left[\frac{\left[\alpha_{\mu} \bar{\mu}+\alpha_{x} \bar{x}_{t}-\left(\alpha_{\mu}+\alpha_{x}\right) p_{t-1}\right]^{2}}{2\left(\alpha_{\mu}+\alpha_{x}\right)}-\frac{\beta^{2}\left(p_{t-1}-v_{t}\right)^{2}}{2 \sigma_{t-1}^{2}}\right]\right\}=1
$$

Solving for $\bar{x}_{t}$ yields

$$
x_{t}^{ \pm}=\frac{1}{\alpha_{x}}\left[\left(\alpha_{\mu}+\alpha_{x}\right) p_{t-1}-\alpha_{\mu} \bar{\mu} \pm \frac{\beta \sqrt{\alpha_{\mu}+\alpha_{x}}}{\sigma_{t-1}}\left(p_{t-1}-v_{t}\right)\right] .
$$

In the case when $E_{i, t}^{f}(U)=E_{i, t}^{c}(U)$, an agent is indifferent between choosing strategies $f$ and $c$ and we assume that she chooses strategy $f$. Then, when $p_{t-1}=v_{t}$, all agents choose strategy $f$. If $p_{t-1} \neq v_{t}$, then the agent chooses strategy $c$ if her signal falls into the interval $\left(x_{t}^{m}, x_{t}^{M}\right)$ and strategy $f$ otherwise, where $x_{t}^{m}=\min \left(x_{t}^{ \pm}\right)$and $x_{t}^{M}=\max \left(x_{t}^{ \pm}\right)$. Therefore, the optimal demand of agent $i$ is determined by

$$
q_{i, t}=\left\{\begin{array}{llc}
q_{i, t}^{f} & \text { if } & x_{i, t} \leq x_{t}^{m} \text { or } x_{i, t} \geq x_{t}^{M} \\
q_{i, t}^{c} & \text { if } & x_{i, t} \in\left(x_{t}^{m}, x_{t}^{M}\right)
\end{array}\right.
$$

Intuitively, when agent's private signal is near the mean fundamental value, the private information of the agent becomes less valuable (in terms of the value function based on the fundamental strategy) and the agent tends to choose momentum strategy which is expected to generate a higher 
utility. However, when agent's private signal is far away from the mean fundamental value, the private information of the agent becomes more valuable and the agent favor the fundamental trading strategy. For convenience, we call agent $i$ fundamentalist if she chooses the fundamental strategy so that $q_{i, t}=q_{i, t}^{f}$ and chartist if she chooses the momentum strategy so that $q_{i, t}=q_{i, t}^{c}$. An agent $i$ chooses to be a fundamentalists if her signal is in the tails of the distribution such that $x_{i, t} \leq x_{t}^{m}$ or $x_{i, t} \geq x_{t}^{M}$, and a chartist otherwise.

Recall that $x_{i, t} \sim N\left(\mu, 1 / \alpha_{x}\right)$. Let

$$
y_{i, t}=\sqrt{\alpha_{x}}\left(x_{i, t}-\mu\right), \quad y_{t}^{ \pm}=\sqrt{\alpha_{x}}\left(x_{t}^{ \pm}-\mu\right),
$$

then from $(8)$

$$
y_{t}^{ \pm}=\frac{1}{\sqrt{\alpha_{x}}}\left[\left(\alpha_{\mu}+\alpha_{x}\right) p_{t-1}-\left(\alpha_{\mu} \bar{\mu}+\alpha_{x} \mu\right) \pm \frac{\beta \sqrt{\alpha_{\mu}+\alpha_{x}}}{\sigma_{t-1}}\left(p_{t-1}-v_{t}\right)\right]
$$

Denote

$$
y_{t}^{m}=\sqrt{\alpha_{x}}\left(x_{t}^{m}-\mu\right), \quad y_{t}^{M}=\sqrt{\alpha_{x}}\left(x_{t}^{M}-\mu\right) .
$$

Then the demand function (9) can be written as

$$
q_{i, t}=\left\{\begin{array}{llc}
q_{i, t}^{f} & \text { if } & y_{i, t} \leq y_{t}^{m} \text { or } y_{i, t} \geq x_{t}^{M} \\
q_{i, t}^{c} & \text { if } & y_{i, t} \in\left(y_{t}^{m}, y_{t}^{M}\right)
\end{array}\right.
$$

in which $x_{i, t}$ in (9) is replaced by $x_{i, t}=\mu+y_{i, t} / \sqrt{\alpha_{x}}$. The standard normal probability density and cumulative probability functions are denoted, respectively,

$$
\phi(x)=\frac{1}{\sqrt{2 \pi}} e^{-x^{2} / 2}, \quad \Phi(x)=\int_{-\infty}^{x} \phi(y) d y .
$$

It then follows from the demand function (11) that the chartists are those whose signals fall into 
$\left(y_{t}^{m}, y_{t}^{M}\right)$. Denote $m_{t}$ the fraction of the chartists, then

$$
m_{t}=\Phi\left(y_{t}^{M}\right)-\Phi\left(y_{t}^{m}\right)
$$

Note that $d m_{t} / d t=\phi\left(y_{t}^{M}\right)-\phi\left(y_{t}^{m}\right)$. As the market consists of fundamentalists and chartists only, the fraction of fundamentalists becomes $1-m_{t}$.

Different from the existing HAMs where the fundamentalists share the same demand function, in this paper, because of the information dispersion, the fundamentalists have different demand functions corresponding to their different signals on the fundamental values. With equation (9) and the notation in (13), the aggregate demand of all the agents is then given by

$$
\begin{aligned}
D_{t}= & \int_{-\infty}^{y_{t}^{m}} \frac{\alpha_{\mu} \bar{\mu}+\alpha_{x} \mu+\sqrt{\alpha_{x}} y_{i, t}-\left(\alpha_{\mu}+\alpha_{x}\right) p_{t-1}}{A \gamma} \phi\left(y_{i, t}\right) d y_{i, t} \\
& +\int_{y^{M}}^{+\infty} \frac{\alpha_{\mu} \bar{\mu}+\alpha_{x} \mu+\sqrt{\alpha_{x}} y_{i, t}-\left(\alpha_{\mu}+\alpha_{x}\right) p_{t-1}}{A \gamma} \phi\left(y_{i, t}\right) d y_{i, t}+m_{t} \frac{\beta\left(p_{t-1}-v_{t}\right)}{A \sigma_{t-1}^{2}} \\
= & \frac{\sqrt{\alpha_{x}}}{A \gamma}\left(\int_{-\infty}^{y_{t}^{m}} y_{i, t} \phi\left(y_{i, t}\right) d y_{i, t}+\int_{y_{t}^{M}}^{+\infty} y_{i, t} \phi\left(y_{i, t}\right) d y_{i, t}\right) \\
& +\left(1-m_{t}\right) \frac{\alpha_{\mu} \bar{\mu}+\alpha_{x} \mu-\left(\alpha_{\mu}+\alpha_{x}\right) p_{t-1}}{A \gamma}+m_{t} \frac{\beta\left(p_{t-1}-v_{t}\right)}{A \sigma_{t-1}^{2}}
\end{aligned}
$$

Using (12), the above demand function can be rewritten as

$$
\begin{aligned}
D_{t}= & \frac{\alpha_{x}}{A \gamma}\left[\frac{1}{\sqrt{2 \pi \alpha_{x}}}\left(e^{-\frac{\left(y_{t}^{M}\right)^{2}}{2}}-e^{-\frac{\left(y_{t}^{m}\right)^{2}}{2}}\right)+\mu\left(1-\left(\Phi\left(y_{t}^{M}\right)-\Phi\left(y_{t}^{m}\right)\right)\right)\right] \\
& +\left[1-\left(\Phi\left(y_{t}^{M}\right)-\Phi\left(y_{t}^{m}\right)\right)\right] \frac{\alpha_{\mu} \bar{\mu}-\left(\alpha_{\mu}+\alpha_{x}\right) p_{t-1}}{A \gamma}+\left[\Phi\left(y_{t}^{M}\right)-\Phi\left(y_{t}^{m}\right)\right] \frac{\beta\left(p_{t-1}-v_{t}\right)}{A \sigma_{t-1}^{2}}
\end{aligned}
$$

or alternatively, using (12) and (13),

$$
D_{t}=\frac{\sqrt{\alpha_{x}}}{A \gamma}\left[\phi\left(y_{t}^{M}\right)-\phi\left(y_{t}^{m}\right)\right]+\left[1-m_{t}\right] \frac{\alpha_{\mu} \bar{\mu}+\alpha_{x} \mu-\left(\alpha_{\mu}+\alpha_{x}\right) p_{t-1}}{A \gamma}+m_{t} \frac{\beta\left(p_{t-1}-v_{t}\right)}{A \sigma_{t-1}^{2}}
$$

To determine the market price, we follow Day and Huang (1990) and assume that a maker maker 
adjusts the price according to

$$
p_{t}=p_{t-1}+\lambda\left(D_{t}+S_{t}\right)
$$

where $\lambda>0$ measures the impact of marginal aggregate demand on the asset price, and $S_{t} \sim$ $N\left(0, \sigma_{s}^{2}\right)$ is an exogenous supply of the risky $\operatorname{asset}^{5}$. The price updating mechanism suggests that the price goes up in the next period if $D_{t}+S_{t}>0$, declines or remains constant otherwise.

In the absence of supply shock (that is $S_{t}=0$ ) and all the agents use the fundamental strategy (that is $m_{t}=0$ ), the market equilibrium price under the Walrasian auctioneer scenario reaches an equilibrium when $D_{t}=0$. Solving for the equilibrium price yields

$$
p^{*}=\frac{\alpha_{x} \mu+\alpha_{\mu} \bar{\mu}}{\alpha_{\mu}+\alpha_{x}}
$$

Note that $\mu$ may not necessarily equal to $\bar{\mu}$ due to the information uncertainty. Therefore the equilibrium price $p^{*}$ may not necessarily equal to the fundamental value $\mu$ even if all agents are acting as fundamentalists. The equilibrium price is above the fundamental value if $\mu<\bar{\mu}$, below the fundamental if $\mu>\bar{\mu}$ and equivalent to the fundamental if $\mu=\bar{\mu}$. The potential difference between the equilibrium price $p^{*}$ and the fundamental value $\mu$ is caused by the imperfect information. In our model, even in the absence of supply shock, there are two origins of asset mispricing, the information uncertainty about the fundamental value and the presence of chartists.

To better understand the dynamics of the market price (14), we now consider a simple deterministic model of (14) when there is no supply shock, $\sigma_{t-1}^{2}=\sigma^{2}$ and $v_{t}=p_{t-2}$. In this case, the price dynamics become

$$
p_{t}=p_{t-1}+\lambda\left[\frac{\sqrt{\alpha_{x}}}{A \gamma} m_{t}^{\prime}+\left(1-m_{t}\right) \frac{\alpha_{\mu} \bar{\mu}+\alpha_{x} \mu-\left(\alpha_{\mu}+\alpha_{x}\right) p_{t-1}}{A \gamma}+m_{t} \frac{\beta\left(p_{t-1}-p_{t-2}\right)}{A \sigma^{2}}\right]
$$

It is easy to see that the fundamental equilibrium price $p^{*}$ defined in (15) is the unique fixed point of (16) and its stability can be characterized by the following proposition.

\footnotetext{
${ }^{5}$ Alternatively, $S_{t}$ can be interpreted as the demand of the noise trader as in Black (1986).
} 
Proposition 1 The fundamental equilibrium price $p^{*}$ of the price dynamics (16) is locally stable if and only if

$$
\lambda \frac{\alpha_{\mu}+\alpha_{x}}{A \gamma}<2
$$

In addition, the loss of stability leads to a flip bifurcation when $\lambda \frac{\alpha_{\mu}+\alpha_{x}}{A \gamma}=2$.

Proposition 1 shows that the stability of the fundamental value is maintained with small precisions of the noisy processes. This implies that less noise in the fundamental information can contribute toward instability of the fundamental value, leading to high price volatility even when the equilibrium is unique. Intuitively, when the dispersion of the fundamental information becomes small, the fundamental information become more accurate but less valuable while the momentum trading strategy becomes more popular among the agents, which then leads to instability and high volatility. This result is consistent with the literature on coordination game with imperfect information such as Angeletos and Werning (2006). Proposition 1 also shows that the price dynamics of such a deterministic model can switch from stable fundamental value to two-period cycle and then to more complicated behavior. Figure 1 illustrates the bifurcation plot of the price with respect to $\gamma$, the speed that price is expected to converge to the fundamental. It shows that, as the speed of the convergence of the expected price to the fundamental in the fundamental strategy increases, the price becomes more stable. This analysis on the deterministic model provides some insight into the price dynamics of the stochastic model to be discussed in the following.

\section{Implications of Trading Heterogeneity}

The model characterizes the endogenous trading heterogeneity among agents when facing information uncertainty. The agent that seeks to maximize her expected utility may choose different strategies simply because either her signal or the market price has changed over time. Note that the two threshold values $x_{t}^{ \pm}$are time varying. Because of the price change, an agent may choose different strategy even if she receives the same signal. As we are interested in the evolution in the market fractions of agents choosing the fundamental and momentum strategies, it is sufficient to 


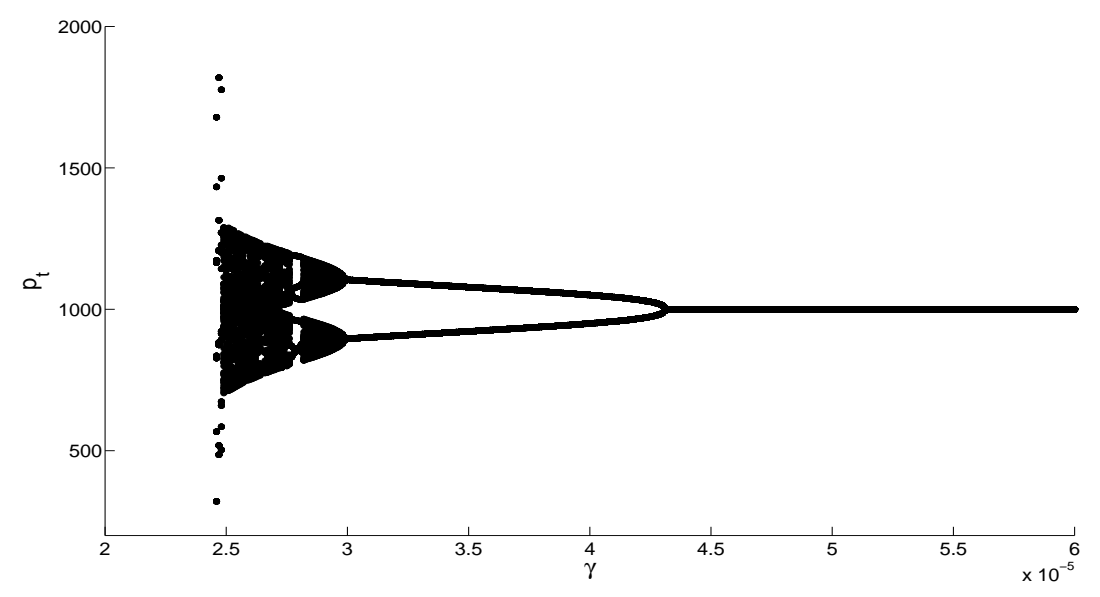

Figure 1: Bifurcation analysis. The price converges to the fixed point $p^{*}=\frac{\alpha_{x} \mu+\alpha_{\mu} \bar{\mu}}{\alpha_{\mu}+\alpha_{x}}$ when $\gamma$ increases to the extent that it satisfies the stability condition that $\lambda \frac{\alpha_{\mu}+\alpha_{x}}{A \gamma}<2$. The parameter used for the bifurcation is $p_{0}=950, p_{1}=954, \sigma_{s}^{2} \equiv 1, \bar{\mu}=\mu=1000, \beta=0.004,1 / \alpha_{u}=4 \times 10^{4}$, $1 / \alpha_{x}=8 \times 10^{4}$ and $\lambda=2.3$.

know the distribution of the signals instead of every agent's signal. Below we illustrate how various factors affect the evolution of the fraction of the chartists, $m_{t}$, over various ranges of prices. Unless specified otherwise, we use the following set of parameters: $\bar{\mu}=\mu=1000, \gamma=0.4, \beta=0.9$, $1 / \alpha_{\mu}=4 \times 10^{4}, 1 / \alpha_{x}=8 \times 10^{4}, \lambda=2.3, p_{t-1}-v_{t}=3, \sigma_{t-1}^{2} \equiv 1, A=1$ and $\sigma_{s}^{2} \equiv 0$.

\subsection{The Impact of Mispricing}

We first examine the impact of mispricing. As shown by Figure 2, the fraction of chartists $m_{t}$ decreases as the price deviates more from the fundamental $\mu$ (for both values in $\left|p_{t-1}-v_{t}\right|$ and $\beta$ ). In another words, agents' choose to the fundamental strategy increase with the degree of mispricing $\left|p_{t-1}-\mu\right|$. In fact, it follows from (10) that

$$
y_{t}^{ \pm}=\frac{1}{\sqrt{\alpha_{x}}}\left[\left(\alpha_{\mu}+\alpha_{x}\right)\left(p_{t-1}-\mu\right)-\alpha_{\mu}(\bar{\mu}-\mu) \pm \frac{\beta \sqrt{\alpha_{\mu}+\alpha_{x}}}{\sigma_{t-1}}\left(p_{t-1}-v_{t}\right)\right]
$$

This, together with (13), implies that $\partial\left(m_{t}\right) / \partial\left(\left|p_{t-1}-\mu\right|\right)<0$, meaning that the fraction of agents choosing the momentum strategy decreases as the mispricing increases. This result is illustrated in Fig. 2 with respect to different price trend $\left|p_{t-1}-v_{t}\right|$ and extrapolation $\beta$ of the momentum 


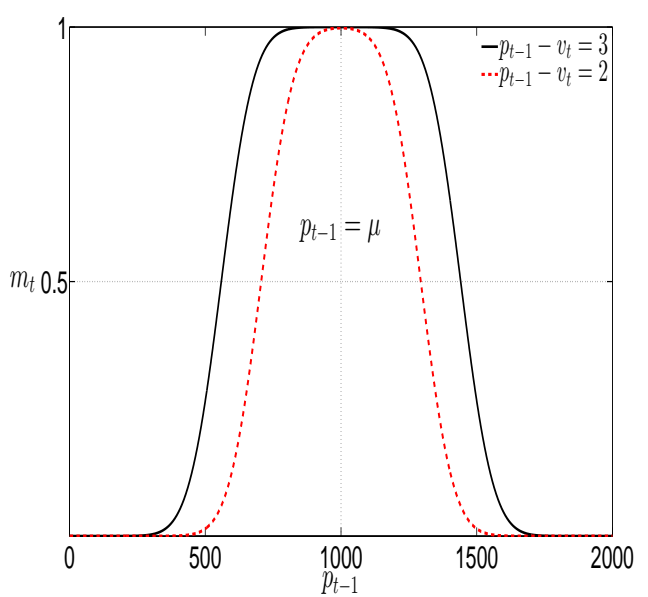

(a)

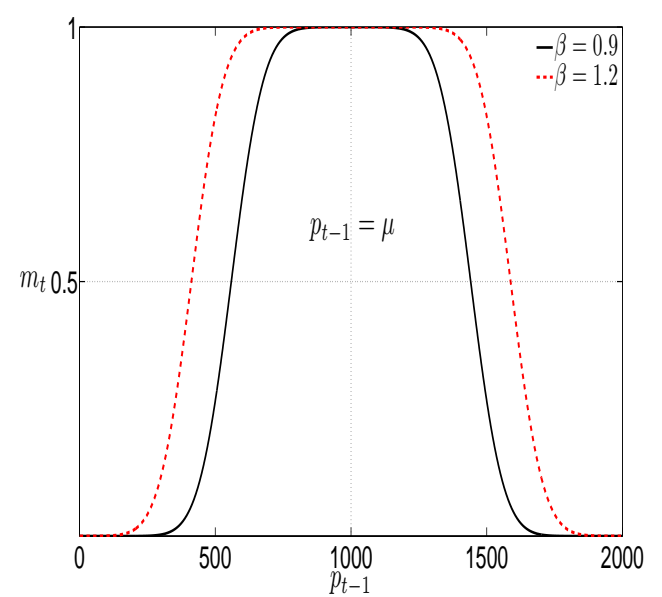

(b)

Figure 2: The effect of mispricing $\left|p_{t-1}-\mu\right|$ and momentum trading on the fraction of the chartists $m_{t}$ with (a) two different price trend $\left|p_{t-1}-v_{t}\right|=3$ and 5; and (b) two different extrapolation $\beta=0.9$ and 1.2.

trading strategy. It shows that $m_{t}$ is close to 1 when the price $p_{t-1}$ is near the fundamental value $\mu=1000$, meaning most of the agents choose the momentum strategy. However, $m_{t}$ decreases as the price $p_{t-1}$ deviates from the fundamental value $\mu$, meaning that more agents choose the fundamental strategy. This suggests that it is unlikely for the price to deviate far away from its value infinitely. All the others being the same, the price reverts towards its fundamental value when it is significantly mispriced, any bubble eventually bursts and recession eventually recovers as significant mispricing trigger agents to choose the fundamental strategy that drive the price towards its fundamental value. Such nonlinear mean-reverting price movements are consistent with De Grauwe et al. (1993), Gaunersdorfer and Hommes (2007), Bauer et al. (2009), Dieci and Westerhoff (2010), among many others. Most studies derive the mean-reverting price behavior by assuming the distribution of fundamentalists and chartists in different market states similar to Figure 2. The model developed in this paper provides a micro-foundation to the endogenize switching of the choices between heterogeneous strategies as a consequence of utility maximization under information friction. ${ }^{6}$

\footnotetext{
${ }^{6}$ We would like to thank the Associate Editor for emphasizing this contribution to the current HAMs.
} 


\subsection{The Impact of Momentum Trading}

We further examine the impact of the market power of momentum trading on the fraction $m_{t}$. Recall from Eq.(7) that all momentum strategy shares the same demand function. The market power of the momentum trading can be measured by $\left|p_{t-1}-v_{t}\right|$ and the extrapolation $\beta$ of the momentum strategy. Based on (9) and (10), an increase in $\left|p_{t-1}-v_{t}\right|$ and $\beta$ enlarges the interval $\left(x_{i}^{m}, x_{i}^{M}\right)$. This means that, as the market power of the momentum trading increases, there are less extreme signals outside of the interval and hence more agents choose the momentum trading strategy, leading to higher $m_{t}$. This result is illustrated in Figure 2. In (a), we compare $m_{t}$ for two different values of $\left|p_{t-1}-v_{t}\right|$. The solid curve represents $m_{t}$ with relatively large $\left|p_{t-1}-v_{t}\right|=3$, while the dashed curve describes $m_{t}$ with relatively small $\left|p_{t-1}-v_{t}\right|=2$. We observe from Figure 2(a) that, given the same $p_{t-1}, m_{t}$ is larger when $\left|p_{t-1}-v_{t}\right|$ is higher. We have the same observation in Figure 2(b) with respect to the extrapolation $\beta$ of the momentum trading.

The above analysis suggests that, on the one hand, the greater the market power of the momentum trading, the higher the market fraction of chartists $m_{t}$ is, which may destabilize the market price, leading to significant mis-pricing. This destabilizing role of the momentum trading is consistent with the current HAMs. On the other hand, based on the previous discussion, a significant mis-pricing motivates more agents to choose the fundamental strategy, driving the market price towards the fundamental value. This endogenous "self-correction" mechanism of the market is very different from the current HAMs where market stability depends exogenously on the balanced activities from the fundamentalists and chartists.

\subsection{Market Fractions under Regime-dependent Reference Price}

Previously we assume $\left|p_{t-1}-v_{t}\right|$ to be exogenously in order to better understand the impact of the market power of momentum trading on market price. In the literature, the reference price $v_{t}$ of the momentum strategy is usually a function of the historical price and can be different over different price range. We follow Huang, et al. $(2010,2012)$ and define $v_{t}$ as regime-dependent reference price. In particular, we divide the price domain $\mathbb{P}=\left[p_{\min }, p_{\max }\right]$ equally into $n$ mutually exclusive 


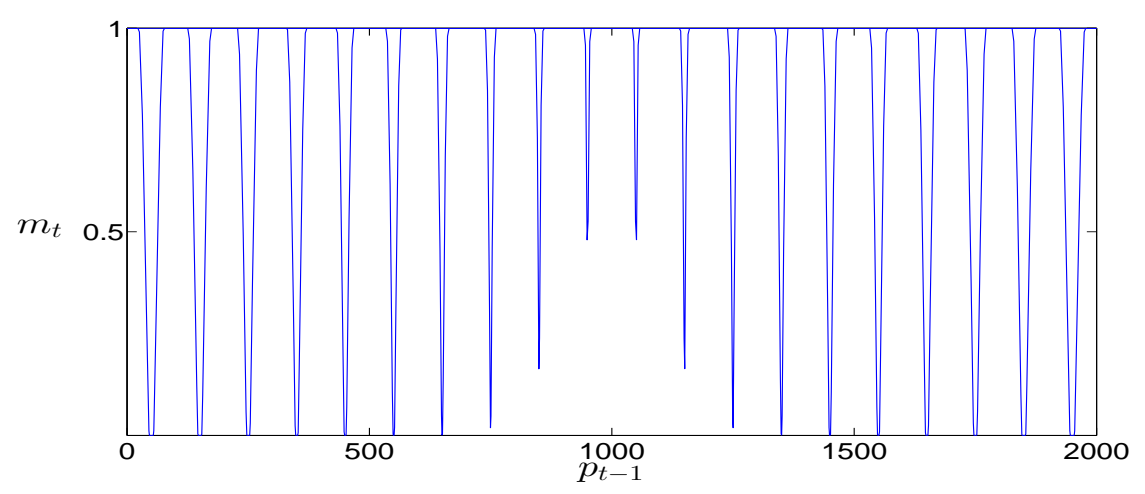

Figure 3: The impact of regime-dependent $v_{t}$ on $m_{t}$.

regimes such that

$$
\mathbb{P}=\cup_{j=1}^{n} \mathbb{P}_{j}=[0, \kappa) \cup[\kappa, 2 \kappa) \cup \cdots \cup[(n-1) \kappa, n \kappa],
$$

where $j \kappa(j=1,2 \ldots, n$.) represents psychological threshold corresponding to different support (resistance) level in the chartist analysis. When $p_{t-1}$ is observed, $v_{t}$ is extrapolated to be in the middle of the trading window that $p_{t-1}$ falls into, that is:

$$
v_{t}=\left(\left\lfloor p_{t-1} / \kappa\right\rfloor+\left\lceil p_{t-1} / \kappa\right\rceil\right) \cdot \kappa / 2
$$

where $\left\lfloor p_{t-1} / \kappa\right\rfloor$ and $\left\lceil p_{t-1} / \kappa\right\rceil$ are the lower and upper bounds of the regime that enclose $p_{t-1}$.

Let $\kappa=100$, based on the regime-dependent $v_{t}$, we plot $m_{t}$ against $p_{t-1}$ in Fig. 3. It shows that, as the price changes, the fraction of chartists $m_{t}$ changes between 1 and 0 frequently. When $p_{t-1}$ is relatively close to the center of the regime $v_{t}$, the market power of the momentum trading is weak and $m_{t}$ moves towards 0 . Otherwise, when $p_{t-1}$ is on the boundary of the regime, $m_{t}$ changes towards 1 . As the price changes from one regime to another, the regime-dependent $v_{t}$ updates accordingly, which changes $m_{t}$ accordingly. Such regime-dependent $v_{t}$ makes agents more sensitive to the price changes and introduces more volatility to $m_{t}$ by enhancing the switching between choosing the two strategies.

As $v_{t}$ is a function of the past price, the price dynamics is essentially one-dimension. Such a model is nonlinear, maybe even chaotic, which is capable of generating rich price dynamic patterns. 


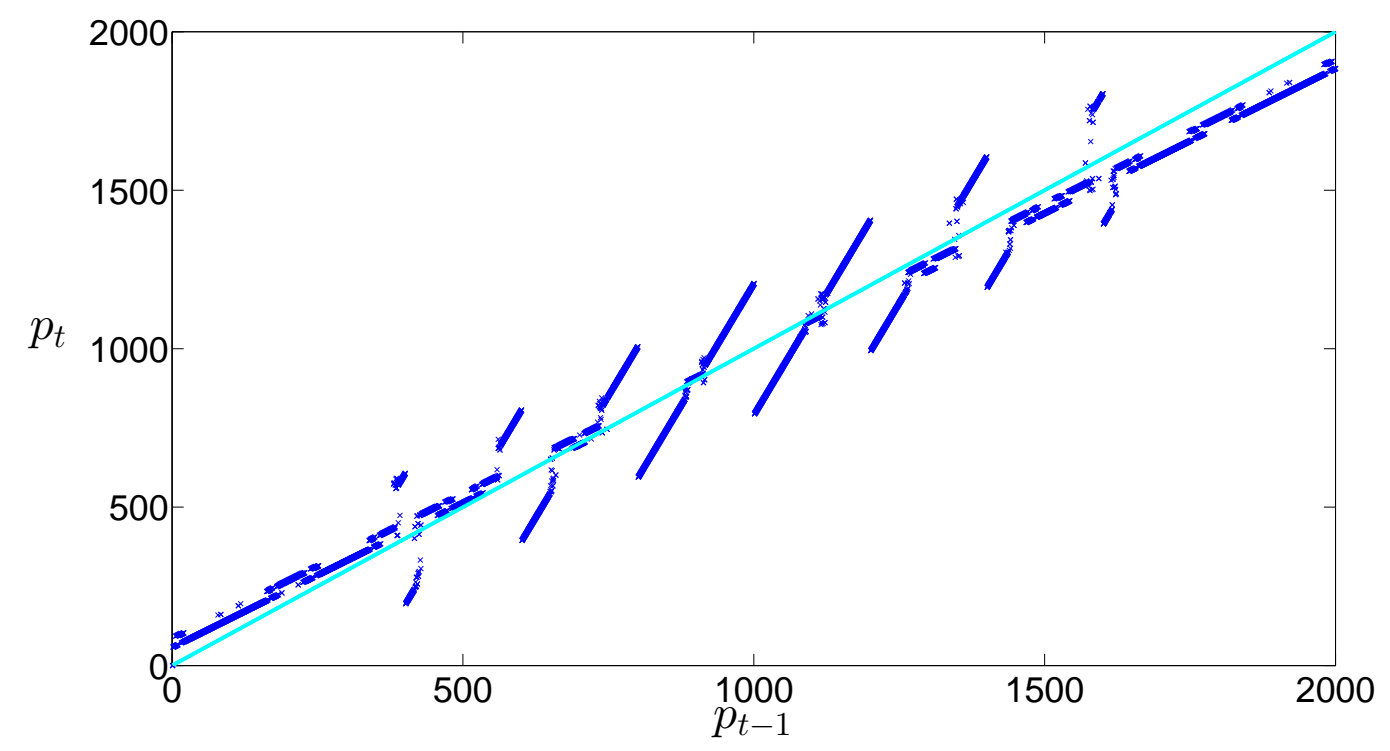

Figure 4: The phase diagram of $p_{t}$ and $p_{t+1}$.

Given the complexity of the model, we illustrate a typical phase diagram of the model in Figure 4, with $\kappa=200, \alpha_{x}=1 / 200, \alpha_{\mu}=1 / 100$ and other parameters given in the standard parameter set. The asterisk-marked curve captures the phase line that maps $p_{t-1}$ to $p_{t}$. It cross the 45 degree line on which $p_{t-1}=p_{t}$ several times, suggesting that there are multiple equilibria. Moreover, when the price is significantly higher than the fundamental value $(\mu=1000)$, the phase line is always below the 45 degree line, which means that the price will subsequently decline when it is sufficiently overpriced. In particular, given any $p_{t-1}$ that is sufficiently higher than the fundamental value, it is observed from Figure 4 that $p_{t}<p_{t-1}$. This is because, when the asset is significantly overpriced, agents cluster almost entirely to be fundamentalists (see Figure 2), which drives the price down towards its fundamental value. Similarly, when the price is significantly lower than the fundamental value, the phase line is above the 45 degree line, indicating that the price will subsequently rebounds when it is sufficiently underpriced. The result is driven by agents clustering to fundamentalists when the asset is significantly underpriced. When market price is not far away from the fundamental value, the trading is dominated by momentum strategy, which increases price fluctuation, indicating by the significant upward piece-wise phase lines when price is around the fundamental vale $(\mu=1000)$. This again illustrates the price fluctuations and endogenous 
self-correction when the market price deviates far away from the fundamental value.

\section{Time Series Properties and the Stylized Facts}

The current literature on HAMs is powerful in reproducing commonly observed financial market abnormalities that cannot be justified by efficient market hypothesis. In this section, we explore the time series properties numerically and in particular focus on whether our HAM with endogenous heterogeneity can also generate the stylized facts in financial time series. We test for the nonnormality, fat tails and volatility clustering in returns. We also examine whether the return series exhibit the power-law distribution and long-memory process (or long-range dependence), which are widely observed in financial markets.

To understand the effect of the stochastic noise, we simulate two price series, one with and one without stochastic supply shock. In the absence of the supply shock, the model is deterministic. A deterministic data generating process is unrealistic given that the price is largely unpredictable, however it provides a good way to understand the performance of the model. Note that these stylized facts are commonly observed across international financial markets over decades while exogenous shocks are random, they are more likely driven by some endogenous price dynamics instead of random shocks. Therefore we are interested in if the deterministic model helps to generate these stylized facts. In general, as shown in $\mathrm{He}$ and $\mathrm{Li} \mathrm{(2007),} \mathrm{it} \mathrm{is} \mathrm{the} \mathrm{interaction} \mathrm{between} \mathrm{the}$ underlying deterministic dynamics and noise that characteristics the realistic time series properties.

To see how well the simulated data matches with the real data in terms of statistical and qualitative properties, we benchmark for each stylized fact based on the daily price of S\&P 500 index from August 28, 2006 to August 7, 2014. The compounded single-period return of the risky asset from $t-1$ to $t$ is defined as $r_{t}=\log \left(p_{t}\right)-\log \left(p_{t-1}\right)$. To differentiate the time series generated from the S\&P 500 index (SP), the simulation based on deterministic model (SD) and the simulation based on stochastic model (SS), we add SP, SD and SS in front of the name of each time series. For example SP $p_{t}, \mathrm{SD} p_{t}$ and $\mathrm{SS} p_{t}$ denote respectively the price of S\&P 500 index, the 
simulation of the deterministic and the stochastic models respectively. Similarly, SP $r_{t}$, SD $r_{t}$ and SS $r_{t}$ denote the log returns based on the three price series SP $p_{t}$, SD $p_{t}$ and SS $p_{t}$.

The set of parameters used to simulate the deterministic and stochastic price is common: $p_{0}=$ $1295.09, \kappa=43.891, \sigma_{s}^{2} \equiv 1$ and the other parameters are the same with the standard parameter set $S: \bar{\mu}=\mu=1000, \gamma=0.4, \beta=0.9,1 / \alpha_{u}=4 \times 10^{4}, 1 / \alpha_{x}=8 \times 10^{4}, A=1$ and $\lambda=2.3{ }^{7}$ The parameter value of $p_{0}$ equals to the first observation of the S\&P 500 sample, and that of $\bar{\mu}$ and $\mu$ is broadly calibrated with the average fundamental value of S\&P 500 calculated based on Gordon growth model. Among the other parameters, $\beta, \gamma, \lambda, \alpha_{u}$ and $\alpha_{x}$, we focus on the two key behavior parameters $\gamma$ and $\beta$ later. We use this standard parameter set throughout the paper, unless specified otherwise. As our purpose is to illustrate if the model has the capability to generate various stylized facts as the conventional HAMs do, we focus on typical deterministic and stochastic simulated price series. A Monte Carlo simulation and sensitivity analysis are conducted to analyze the impact of the two key behavior parameters $\beta$ and $\gamma$ later.

\subsection{Time Series Properties}

Before examining the stylized facts, we first present some time series properties on price and return. Figure 5 plots time series of the prices and returns of the index and a typical simulation. Although the price trajectories for the three price series of (a) the index $S P p_{t}$, (c) the deterministic model $S D p_{t}$ and (e) the stochastic model $S S p_{t}$ look quite different, we do observe commonly that the prices move up and down with occasional bubbles and crashes. For the simulated prices, even though the fundamental value is constant, the price are quite volatile, suggesting the presence of excess volatility. The results are consistent with the empirical evidence documented in Ebrahim and Mathur (2001), Taylor (2007) and early HAMs for example Lux (1995). The commonly shared features by the corresponding three return series in (b), (d) and (f) are: (i) the return moves around zero; (ii) high and low volatility tend to cluster together, suggesting that small (large) changes in returns tend to be followed by small (large) changes in return; and (iii) returns are generally more

\footnotetext{
${ }^{7}$ Note that the parameter $\sigma_{s}^{2}$ is abandoned when simulating the deterministic price.
} 


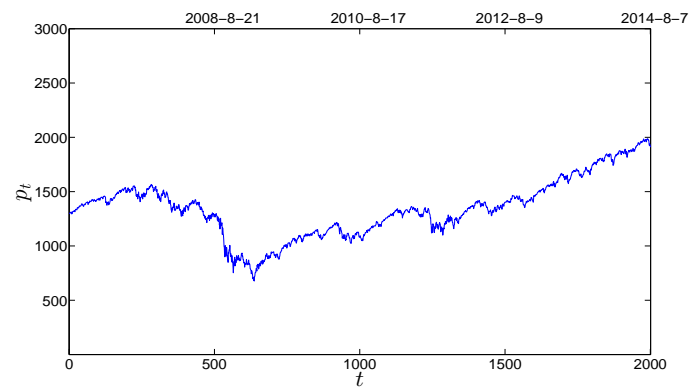

(a) SP $p_{t}$

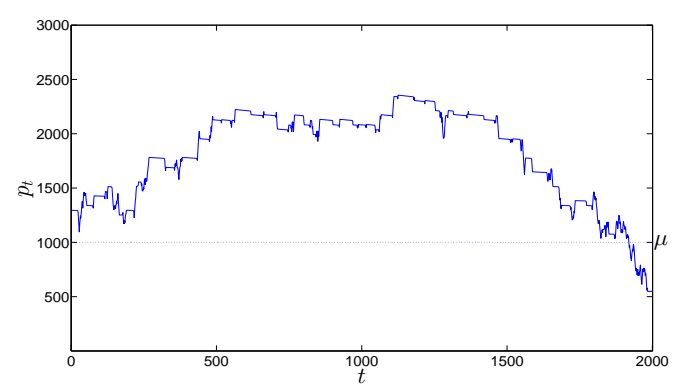

(c) $\mathrm{SD} p_{t}$

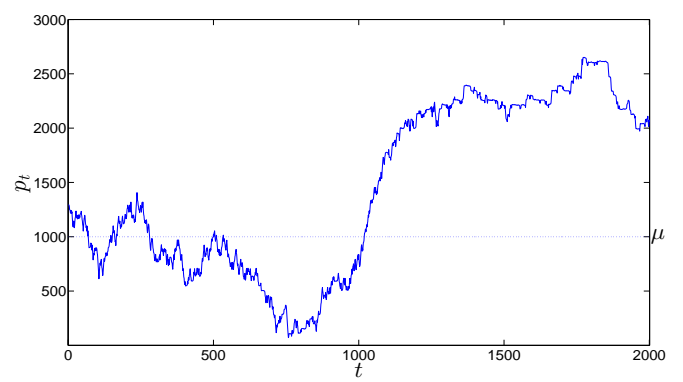

(e) $\mathrm{SS} p_{t}$

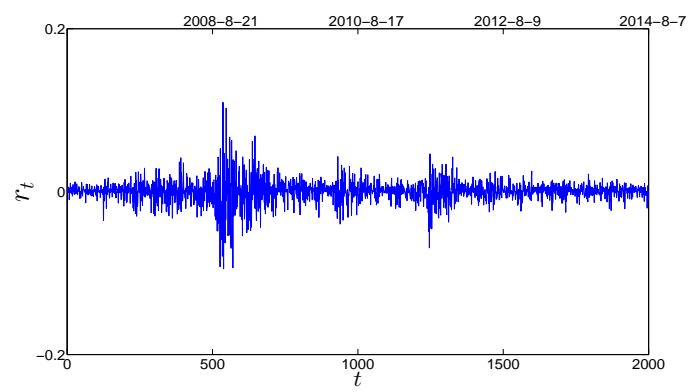

(b) SP $r_{t}$

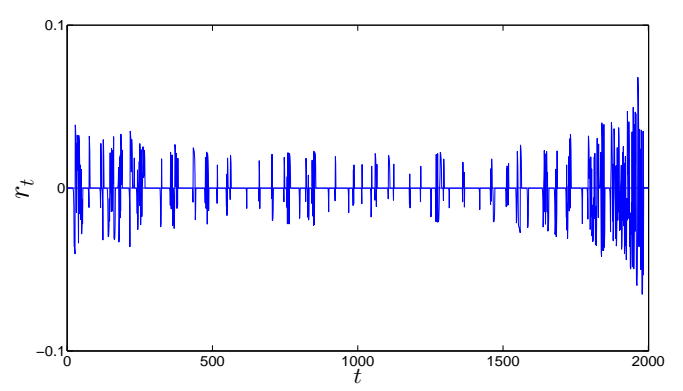

(d) SD $r_{t}$

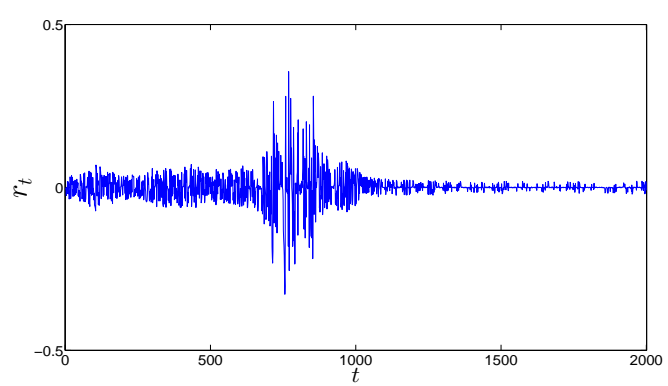

(f) $\mathrm{SS} r_{t}$

Figure 5: The time series of prices and returns on the S\&P500 index (top panel), the simulation from the deterministic model (middle panel), and the simulation from the stochastic model (bottom panel). 
volatile when the price declines. The results suggest that simulations based on our model share some of the time series properties of the real return patterns documented in existing literature.

Table 1 summarizes the statistical properties of returns. In line with the S\&P 500 index, returns generated from the deterministic and stochastic models have negative skewness and high kurtosis. The negative skewness suggests that extremely negative returns are more likely to happen than extremely positive returns. The large kurtosis suggests the presence of fat tails, that is, the extreme returns appear more frequently than what are predicted by the normal distribution. These features suggests that the returns are not normal. This is further supported by p-value of the Jarque-Bera test, which rejects the null hypothesis that either $\mathrm{SP} r_{t}$, or SD $r_{t}$ or SS $r_{t}$ is normally distributed.

Table 1: Summary Statistics of Returns. This table reports the summary statistics, including mean, standard deviation (sd), skewness, kurtosis, and the p-value of the Jarque-Bera test, of the return series of S\&P500 index ( $\mathrm{SP} r_{t}$ ), the simulated deterministic model (SD $r_{t}$ ) and the simulated stochastic model $\left(\mathrm{SS} r_{t}\right)$.

\begin{tabular}{llllllll}
\hline & mean & sd & min & max & skewness & kurtosis & p-value \\
\hline SP $r_{t}$ & 0.000 & 0.014 & -0.095 & 0.110 & -0.325 & 12.525 & 0.000 \\
SD $r_{t}$ & -0.000 & 0.012 & -0.065 & 0.068 & -0.347 & 8.515 & 0.000 \\
SS $r_{t}$ & 0.000 & 0.042 & -0.328 & 0.355 & -0.211 & 19.327 & 0.000 \\
\hline
\end{tabular}

The above results suggest that the model is able to generate realistic time series observed in the S\&P500. They are however very sketchy and basic. To explore further the statistics of the model and compare with the S\&P500 index, we conduct econometric tests to study the degree of volatility clustering, the heaviness of the tails, and the distribution of returns. In paricular, we examine the short-term and long-term features of the price movements and study the long-memory process and long-range dependence.

\subsection{The Power Law of Returns}

Both theoretical and empirical evidence suggests that the tail distribution of return is well approximated by the power law (Gabaix et al., 2003, 2006). In particular the distribution of returns is 
Table 2: The power law of returns. For each return series in SP $r_{t}, \mathrm{SD} r_{t}$ and SS $r_{t}$, we estimate $\ln P\left(\left|\frac{r_{t}-\bar{r}}{s d}\right|>X\right)=-\zeta \ln X+b$, where $\zeta \simeq 3$ is the Pareto exponent. This table reports the estimated $\zeta \simeq 3$, the number of observations $\mathrm{N}$ and the $R^{2}$.

\begin{tabular}{llll}
\hline & $\mathrm{SP} \ln P\left(\left|\frac{r_{t}-r}{s d}\right|>X\right)$ & $\mathrm{SD} \ln P\left(\left|\frac{r_{t}-r}{s d}\right|>X\right)$ & $\mathrm{SS} \ln P\left(\left|\frac{r_{t}-r}{s d}\right|>X\right)$ \\
\hline$\zeta$ & $2.981^{* * *}$ & $3.086^{* * *}$ & $2.681^{* * *}$ \\
& $(14.649)$ & $(12.924)$ & $(12.988)$ \\
$N$ & 55 & 55 & 55 \\
$R^{2}$ & 0.799 & 0.756 & 0.758 \\
\hline
\end{tabular}

found to decay according to

$$
P\left(\left|\frac{r_{t}-\bar{r}}{s d}\right|>X\right) \sim X^{-\zeta}
$$

where $\zeta \simeq 3$ is the tail or Pareto exponent, $\bar{r}$ and $s d$ are the mean and standard deviation of the returns $r_{t}, \frac{r_{t}-\bar{r}}{s d}$ is the normalized return, and $\sim$ denotes asymptotic equality up to numerical constants. In Gabaix $(2003,2006)$, the estimation result $\zeta \simeq 3$ is obtained by letting $X$ take a range of values and estimate with ordinary least squares (OLS) the logarithmal of Eq.(20), that is,

$$
\ln P\left(\left|\frac{r_{t}-\bar{r}}{s d}\right|>X\right)=-\zeta \ln X+b
$$

We follow the same methodology to test whether the simulated returns exhibit such a powerlaw distribution. In particular, we let $X=0.1,0.2, \ldots 0.55$ and calculate $P\left(\left|\frac{r_{t}-\bar{r}}{s d}\right|>X\right)$ for each realization of $X$ for each of SP $r_{t}, \operatorname{SD} r_{t}$ and $\operatorname{SS} r_{t}{ }^{8}$. Based on these observations of $X$ and $P\left(\left|\frac{r_{t}-\bar{r}}{s d}\right|>\right.$ $X$ ), we estimate Eq.(21) with OLS for each return series. The estimation results are reported in Table 2. It shows that, like the tail distribution of SP $r_{t}$, both SD $r_{t}$ and SS $r_{t}$ have a power exponent $\zeta$ of around 3. The fact that estimation coefficients of $\zeta \simeq 3$ confirms the presence of power-law distribution in both the index and simulated return series.

\footnotetext{
${ }^{8}$ If we further increases $X$ above 0.55 , then $P\left(\left|\frac{r_{t}-\bar{r}}{s d}\right|>X\right)=0$ for the time series of SD $r_{t}$. For the other two series, the result that the estimated coefficient $\zeta$ is approximately 3 remains robust even after we increase $X$ as long as $P\left(\left|\frac{r_{t}-\bar{r}}{s d}\right|>X\right)>0$.
} 


\subsection{Volatility Clustering, Long Memory, and Leverage Effect}

As documented in Cont (2001), the autocorrelations function (ACF) of returns are insignificant but different measures of volatility, such as absolute or square returns, exhibit positive and persistent ACFs. We report the ACFs in Figure 6. The trivial ACFs of returns suggest that past return is not necessarily informative for future return. The significantly positive ACFs of volatility measures imply that periods of quiescence and turbulence tend to cluster together. Panels (a)-(c) of Figure 6 demonstrate these characteristics of volatility clustering by piloting ACFs as a function of the number of lags. For the S\&P 500, deterministic and stochastic simulations, there are no significant and decaying ACFs in return (apart from the first lag), but the ACFs of the absolute returns and squared returns are relatively large and persistent even after 100 lags.

To see how persistent the volatility is, we follow Cont (2001) to estimate the following power component in the ACFs of absolute returns:

$$
\operatorname{corr}\left(\left|r_{t+q}\right|,\left|r_{t}\right|\right) \simeq \varsigma / q^{d}
$$

where $q$ is the number of lags, $\varsigma$ is a parameter that captures the ACF of absolute returns with lag one, and $d$ is the power exponent that captures how fast the ACFs decay. For each of the time series of returns, we first obtain the ACFs of the absolute returns for $q=1,2, \ldots 200$, and then estimate Eq.(22) using nonlinear least squares (NLS). Let SP $\operatorname{corr}\left(\left|r_{t+q}\right|,\left|r_{t}\right|\right), \mathrm{SD} \operatorname{corr}\left(\left|r_{t+q}\right|,\left|r_{t}\right|\right)$ and SS $\operatorname{corr}\left(\left|r_{t+q}\right|,\left|r_{t}\right|\right)$ denote the ACFs of absolute returns of SP $r_{t}, \mathrm{SD} r_{t}$ and $\mathrm{SS} r_{t}$ respectively. Table 3 presents the estimation results. We find that the ACFs of absolute returns of S\&P 500, deterministic and stochastic simulations, decay with exponents of $0.368,0.417$ and 0.205 respectively. The results are roughly consistent with the empirical evidence that $d$ generally falls into $[0.2,0.4]$ (see Cont, 2001).

Long memory and long range dependence are synonymous notions that are used interchangeably in the literature. Volatility clustering is an indicator of long memory but it does not necessarily lead to long memory. Following the standard definition in Tsay (2010, Chapter 2) and Campbell, 


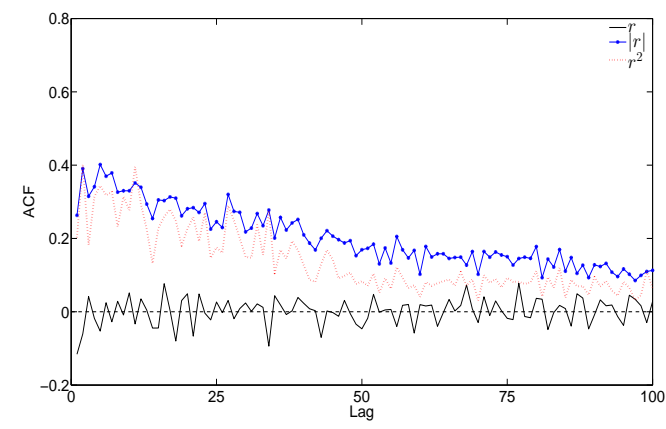

(a) ACFs: SP

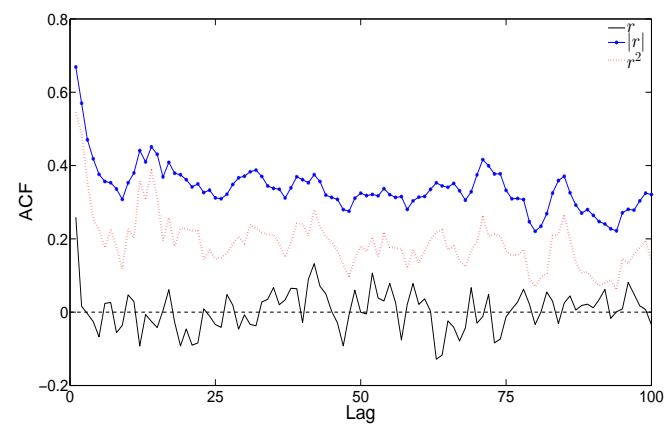

(c) ACFs: SS

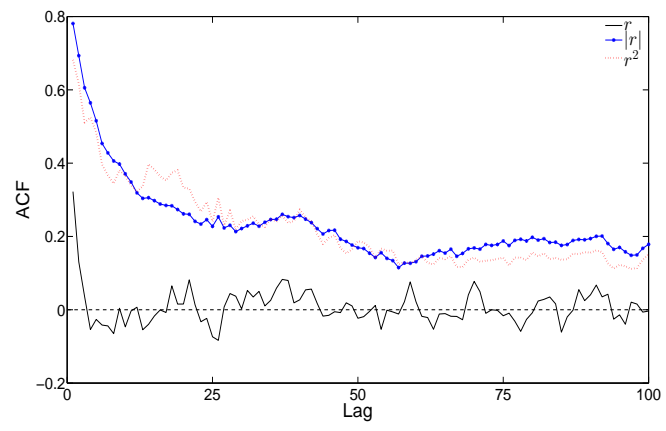

(b) ACFs: SD

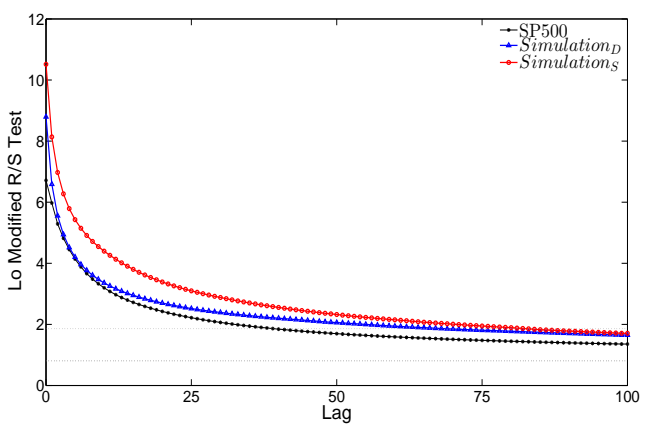

(d) Long Memory

Figure 6: Volatility clustering and Long Memory. Panel (a) plots the autocorrelations function (ACFs) of returns (solid line), absolute returns (asterisk marked line) and squared returns (dotted line) for S\&P500 index against the number of lag. Panel (b) and (c) plots similar statistics based on returns of simulation from deterministic and stochastic model respectively. Panel (d) plots the Lo modified R/S statistic of the absolute returns.

Table 3: Persistence of ACFs of absolute returns. For each return series in SP $r_{t}$, SD $r_{t}$ and SS $r_{t}$, we estimate $\operatorname{corr}\left(\left|r_{t+q}\right|,\left|r_{t}\right|\right) \simeq \varsigma / q^{d}$ with nonlinear least squares and report $\varsigma$ and $d$.

\begin{tabular}{llll}
\hline & SP $\operatorname{corr}\left(\left|r_{t+q}\right|,\left|r_{t}\right|\right)$ & SD $\operatorname{corr}\left(\left|r_{t+q}\right|,\left|r_{t}\right|\right)$ & SS corr $\left(\left|r_{t+q}\right|,\left|r_{t}\right|\right)$ \\
\hline \hline$d$ & $.368^{* * *}$ & $.417^{* * *}$ & $.205^{* * *}$ \\
& $(21.015)$ & $(51.367)$ & $(23.461)$ \\
$\varsigma$ & $.605^{* * *}$ & $.916^{* * *}$ & $.658^{* * *}$ \\
$N$ & & & \\
$N$ & 200 & 200 & 200 \\
$R^{2}$ & .866 & .966 & .974 \\
Root MSE & .058 & .037 & .046 \\
\hline
\end{tabular}


Lo and MacKinlay (1997, Chapter 2), the time series $x_{t}$ is said to be long-memory ${ }^{9}$ if

$$
(1-L)^{d} x_{t}=\varepsilon_{t}
$$

where $L$ is the lag operator, $d \in(-0.5,0.5)$ is the memory parameter that measures the extent of the memory or long range dependence in $\left\{x_{t}\right\}$, and $\left\{\varepsilon_{t}\right\}$ is a white noise series. The long-memory time series $x_{t}$ is said to be fractionally differenced of order $d$. It is neither stationary, where the ACF declines exponentially, nor is it a unit root, where the ACF decays linearly. Such a longmemory process is characterized with small and slowly-decayed ACFs. The decay of ACFs in long-memory process is much faster than in the unit root process for the first lag, but slower for larger lags. When $d \in(-0.5,0.5)$ and $q \rightarrow \infty$, the ACFs of $\left\{x_{t}\right\}$ fades away at a polynomial rate as the lag increases such that:

$$
\operatorname{corr}\left(x_{t}, x_{t+q}\right) \backsim f(q) / q^{1-2 d},
$$

where $f(q)$ is any slowly varying function at infinity, verifying $f(a q) / f(q) \rightarrow 1$ for any $a>0$, as the number of lag $q \rightarrow \infty$.

As documented in Christensen and Nielsen (2007), Christensen, et al. (2010), Bollerslev, et al. (2013), Rossi and Santucci de Magistris (2013), among many others, the stock market volatility has long memory. The phenomenon of volatility clustering discussed above is a necessary but not sufficient condition for the existence of long memory. We now formally test the existence of long memory in the volatility measured by the time series of $\left|r_{t}\right|$. The methodology that we apply is the range over standard deviation or R/S statistic modified by Lo (1991) that corrects for the effects of short-range dependence ${ }^{10}$.

We test for the null hypothesis that there is no long memory if the estimated R/S statistic falls out of the critical interval. The result rejects the null hypothesis and provides evidence that the time series is long-memory. As the Lo modified R/S statistic does not provide a criteria for the

\footnotetext{
${ }^{9}$ Baillie (1996) provides an extensive survey on the definition of long memory.

${ }^{10}$ The other commonly used methodologies is the semiparametric estimator of log periodogram (LP) regression, represented by Geweke and Porter-Hudak (1983), Phillips (2007) and Robinson (1995).
} 


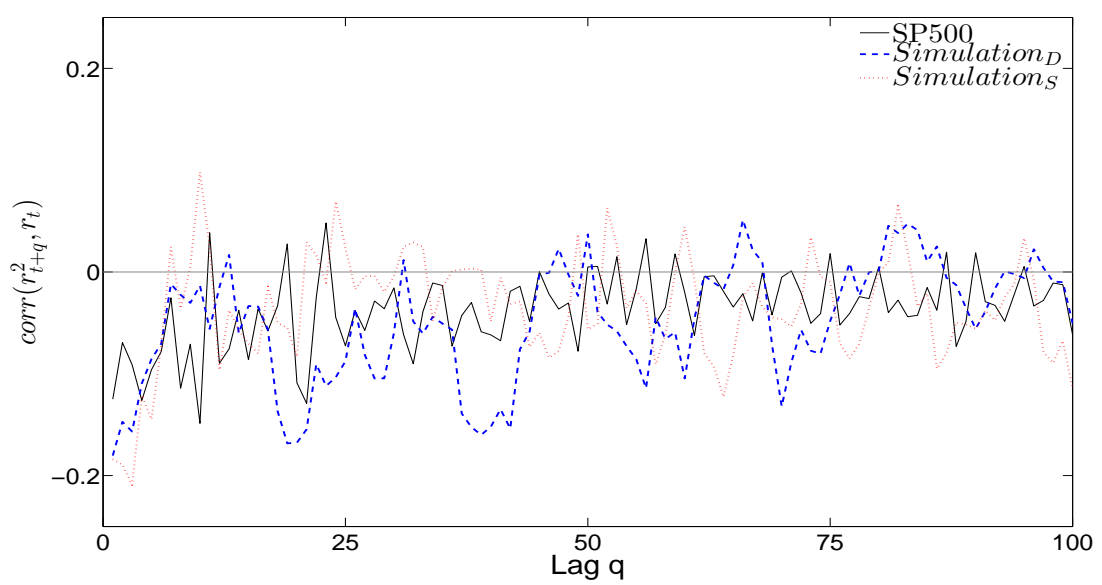

Figure 7: The leverage effect. This figure plots the degree of leverage effect measured by $\operatorname{corr}\left(r_{t+q}^{2}, r_{t}\right)$.

selection of the optimal lag and the R/S statistic maybe sensitive to the selection of lags. We report the R/S statistic for lags ranging from 1 to 100 and graph it in Panel (d) of Figure 6. When the number of lags is not too large, i.e. $q<30$, the Lo modified R/S statistic for SP $\left|r_{t}\right|$, SD $\left|r_{t}\right|$ and SS $\left|r_{t}\right|$ all fall out of the $95 \%$ critical interval $[0.809,1.862]$, which suggests the existence of long memory in both the real and simulated absolute return series.

Other than volatility clustering and long memory, we also observe from Fig. 5 that volatility tends to become higher as price declines and lower as the price rises. The negative correlation between volatility and returns is called leverage effect or volatility asymmetry (Bouchaud, et al., 2001 and Pagan, 1996). Represent the volatility with absolute returns, for each of the three return series, we calculate $\operatorname{corr}\left(r_{t+q}^{2}, r_{t}\right)$ for $q=0,1,2, \ldots 100$. Figure 7 plots $\operatorname{corr}\left(r_{t+q}^{2}, r_{t}\right)$ against the number of lag $q$ for each return series. It shows that when the number of lag is small, i.e. $q<7$, the relation between volatility and returns is negative for each of the three time series of return. This suggests that negative returns are associated with higher contemporary volatility and followed by higher volatility in the short-term. This implies that the model is able to capture the leverage effect observed in financial markets. 


\subsection{Monte Carlo Analysis}

In this section, we conduct a Monte Carlo analysis on the robustness of the results based on the simulations of the stochastic model. We run 1,000 simulations using the same parameter set. In each simulation, the exogenous supply shock $S_{t}$ is normally distributed around 0 with a variance of 1 . The realization of $S_{t}$ takes different random values in the 1000 repeated simulations. ${ }^{11} \mathrm{We}$ repeat the previous statistical analysis for each valid simulation and summarize the results in Table 4. The last column of Table 4 report these statistics based on the S\&P 500 index. Based on the mean of these statistics, it remains robust that the stochastic simulation matches with the S\&P 500 in terms of the first to fourth moments, power-law distribution, volatility clustering and long memory. Looking at the 5\% confidence interval enclosed by p5 and p95 values, we observe that a large proportion of our simulation matches with the S\&P $500 .^{12}$

Table 4: Monte Carlo Analysis. This table reports the summary statistics, including mean, standard deviation (sd), minimum value (min), median value (Med), maximum value (Max), 5th percentile (p5), and 95th percentile (p95), of the various measures of stylized facts in the first column based on 634 different time series of returns. The mean, sd, skewness, kurtosis are the first, second, third and fourth moments of returns respectively. $\zeta$ is the power component in $\ln P\left(\left|\frac{r_{t}-\bar{r}}{s d}\right|>X\right)=$ $-\zeta \ln X+b, d$ is a measure of how fast the autocorrelation function decays, R/S statistic is the Lo-modified test of long-range dependence. The last column reports the corresponding statistics for S\&P 500.

\begin{tabular}{lllllllll}
\hline & Mean & sd & Min & Med & Max & p5 & p95 & SP500 \\
\hline mean & 0.000 & 0.000 & -0.002 & 0.000 & 0.000 & -0.001 & 0.000 & 0.000 \\
sd & 0.021 & 0.012 & 0.006 & 0.018 & 0.071 & 0.009 & 0.047 & 0.014 \\
skewness & 0.036 & 0.218 & -3.044 & 0.025 & 2.168 & -0.102 & 0.249 & -0.325 \\
kurtosis & 6.348 & 9.529 & 2.124 & 3.997 & 126.487 & 2.541 & 18.437 & 12.525 \\
$\zeta$ & 2.929 & 0.435 & 1.542 & 2.956 & 3.942 & 2.124 & 3.575 & 2.981 \\
$d$ & 0.299 & 0.130 & 0.073 & 0.283 & 1.094 & 0.123 & 0.522 & 0.368 \\
R/S statistic & 6.047 & 1.518 & 2.064 & 5.966 & 10.096 & 3.698 & 8.711 & 6.716 \\
\hline
\end{tabular}

\footnotetext{
${ }^{11}$ The simulation may lead to non-positive price that is not realistic. We drop the simulation that generates any non-positive price, which may explain the positive skewness on average in Table 4. Eventually, we include in our sample 634 valid simulations, each of which contains 2000 observations on returns.

${ }^{12}$ This proportion increases further in the $90 \%$ confidence interval (not reported for space constraint). Among the 634 simulated time series of returns, we find 521 (or 82\%) of them have a kurtosis greater than 3, 512 (81\%) of them have a p-value of Jarque-Bera normality test less than 5\%, $356(56 \%)$ of them have a $d$ that falls into [0.2,0.4], and $634(100 \%)$ of them have R/S statistic that fall out of $[0.809,1.862]$ when the number of lag is 2 .
} 

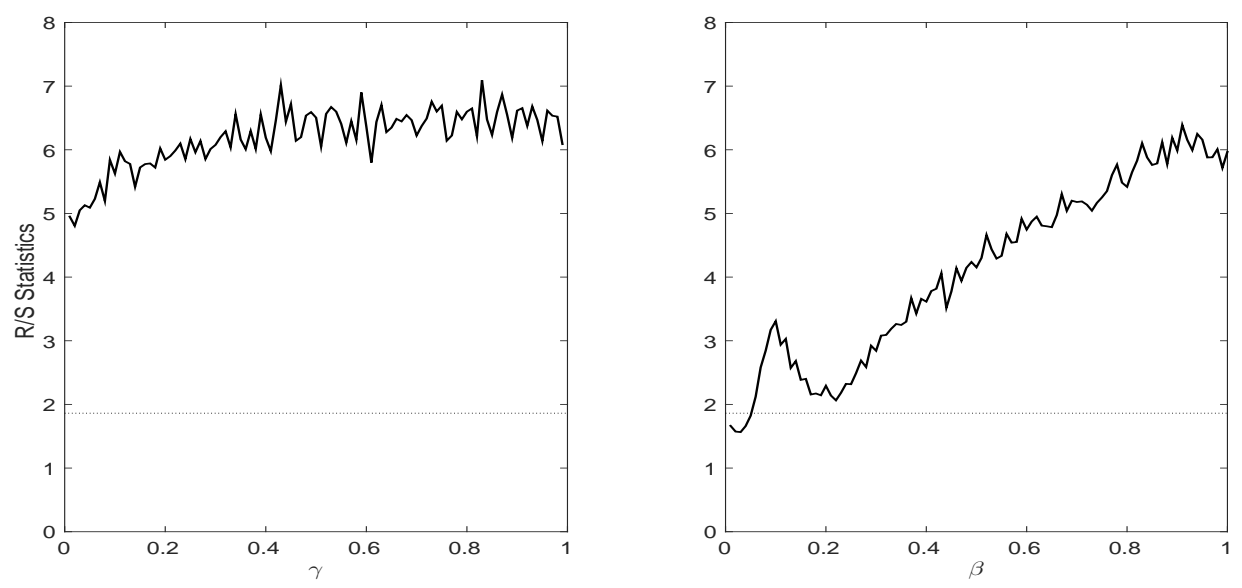

Figure 8: The R/S statistics for $\gamma$ (on the left panel) and $\beta$ (on the right panel).

\subsection{Sensitivity Analysis}

We are also interested in how the stylized facts depends on the choice of behavior parameters of the model, in particular, the mean reverting parameter $\gamma$ of the fundamental strategy and the extrapolation parameter $\beta$ of the momentum strategy. ${ }^{13}$ We first focus on the R/S statistics since long memory is found to be the most challenging one among those stylized facts. With the chosen set of parameters, we vary $\gamma$ and $\beta$, respectively, from 0.01 to 1 with an interval of 0.01 and run 100 simulations for each value. For each simulated time series, we calculate its R/S statistics. For each parameter value, we then calculated the average R/S statistics of the 100 simulated time series and report the results in Figure 8, illustrating the relation between R/S statistics and $\gamma$ on the left panel and $\beta$ on the right panel. The results show the evidence of long memory in returns for all values of $\gamma$ and $\beta$ (except when $\beta \leq 0.05$ ); all the $\mathrm{R} / \mathrm{S}$ statistics are greater than the cutoff value 1.862.

We then examine the impact of $\gamma$ and $\beta$ on volatility clustering, measured by the significant decaying ACFs. For illustration, we choose three representative values for each of $\gamma$ and $\beta$. For each parameter value, we calculate the average ACFs of 100 simulated returns series for all lags

\footnotetext{
${ }^{13}$ The sensitivity analysis in this section means to provide additional evidence on the robustness of the results presented above and to offer some implications on the relation between key parameters and various statistics and stylized facts. Note however due to the large number of parameter sets, the nonlinear and complexity nature of the underlying deterministic model, one should be careful in extending the relation between parameter values and various statistics documented in this section.
} 

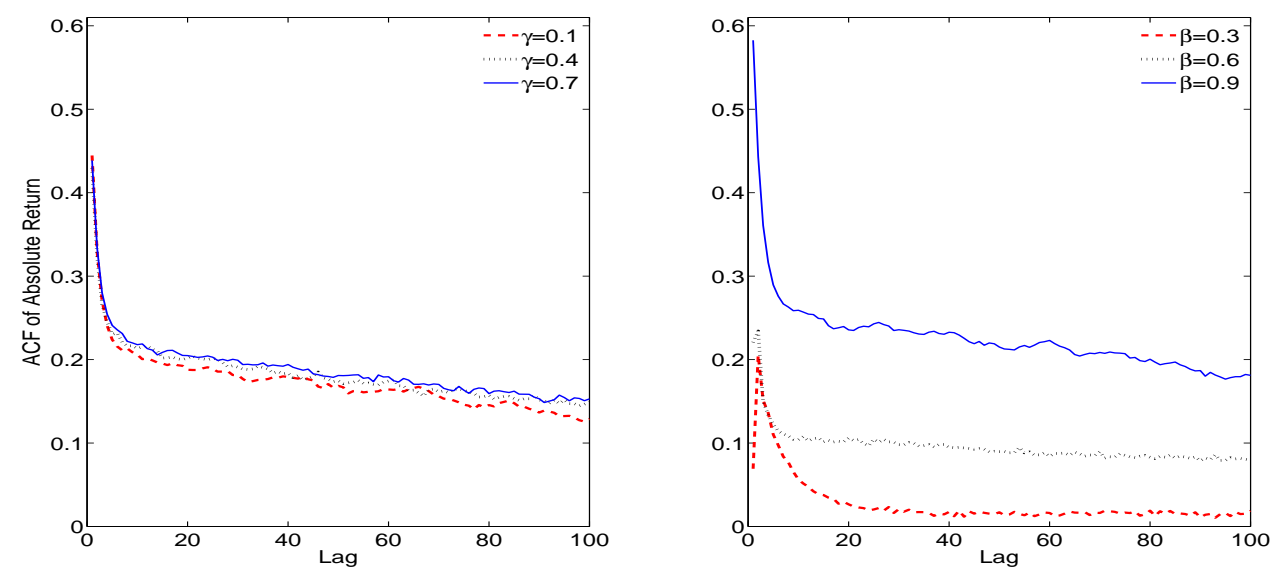

Figure 9: The autocorrelation functions (ACFs) of absolute returns for $\gamma=0.1,0.4,0.7$ (on the left panel) and $\beta=0.3,0.6,0.9$ (on the right panel).

from 1 to 100 and report the result in Figure 9 for $\gamma$ on the left panel and $\beta$ on the right panel. For $\gamma=0.1,0.4$ and 0.7 , the left panel shows that the decaying pattern of the ACFs are very similar, indicating that the volatility clustering is not very sensitive to the mean reverting speed $\gamma$. However, the ACFs patters for $\beta=0.3,0.6$ and 0.9 are very different. It appears that the stronger the extrapolation parameter of the momentum trading $\beta$ is, the higher the ACFs of absolute returns (similar evidence is found if we calculate the ACFs of squared returns), indicating that an increasing in momentum trading enhances volatility clustering.

\section{Conclusion}

Heterogeneity and bounded rationality are two key characteristics of financial markets. Based on complete information and some heuristic assumptions, the existing heterogeneous agent models (HAMs) have been successful in explaining financial market abnormality and stylized facts of financial data and outperforming random walk and many conventional models. This paper contributes to the development of this literature by endogenizing heterogeneity of agents' trading when agents face information uncertainty. Because of the information dispersion, agents that seek to maximize their utility may choose different trading strategies in response to changing market 
environment, which generates cross-sectional trading heterogeneity among agents. The choices of strategies can vary over time due to changes in their private signals and market prices. Consequently, both cross-sectional and time-varying trading heterogeneity arise endogenously. This provides a micro-foundation to the switching mechanism widely documented in the current HAMs. The model is able to generate transitions between bubbles and recessions and to match the real data pretty well with respect to the cubic-law distribution in returns, volatility clustering and long memory, which are commonly observed properties in financial market returns and volatility.

\section{Acknowledgements}

We would like to thank three anonymous reviewers, an anonymous associate editor and the editor Nicolaas J. Vriend for their valuable comments and suggestions. The authors retain sole responsibility for any remaining errors. Financial support from the Australian Research Council under the Discovery Grant (DP130103210), the Quantitative Finance Research Center (QFRC) at UTS, and Hong Kong University Grant Committee under General Research Fund (CUHK14601715) is gratefully acknowledged.

\section{Appendix: Proof of Proposition 1}

First of all, note that at the steady state equilibrium price $p^{*}$, we have $y_{t}^{m}=y_{t}^{M}=0, m_{t}=0$ and $m_{t}^{\prime}=0$. Let $q_{t}=p_{t-2}$, then (16) can be written as

$$
\left\{\begin{array}{l}
p_{t}=p_{t-1}+\lambda D\left(p_{t-1}, q_{t-1}\right) \\
q_{t}=q_{t-1}
\end{array}\right.
$$

where

$$
D\left(p_{t-1}, q_{t-1}\right)=\frac{\sqrt{\alpha_{x}}}{A \gamma} m_{t}^{\prime}+\left(1-m_{t}\right) \frac{\alpha_{\mu} \bar{\mu}+\alpha_{x} \mu-\left(\alpha_{\mu}+\alpha_{x}\right) p_{t-1}}{A \gamma}+m_{t} \frac{\beta\left(p_{t-1}-q_{t-1}\right)}{A \sigma^{2}}
$$


Note that at the stead state $p_{t}=p^{*}$ and $q_{t}=p^{*}$, we have

$$
\frac{\partial D\left(p_{t-1}, q_{t-1}\right)}{\partial p_{t-1}}=-\frac{\alpha_{\mu}+\alpha_{x}}{A \gamma}, \quad \frac{\partial D\left(p_{t-1}, q_{t-1}\right)}{\partial q_{t-1}}=0
$$

This leads to the two eigenvalues:

$$
\Gamma_{1}=0, \quad \Gamma_{2}=1-\lambda \frac{\alpha_{\mu}+\alpha_{x}}{A \gamma}
$$

Therefore $\left|\Gamma_{2}\right|<1$ if and only if $0<\lambda \frac{\alpha_{\mu}+\alpha_{x}}{A \gamma}<2$. In addition, when $\lambda \frac{\alpha_{\mu}+\alpha_{x}}{A \gamma}=2$, we have $\Gamma_{2}=-1$, leading to a flip bifurcation. This completes the proof. 


\section{References}

[1] Alfarano, S., Lux, T. \& Wagner, F. (2008). Time variation of higher moments in a financial market with heterogeneous agents: An analytical approach. Journal of Economic Dynamics and Control 32(1): 101-136.

[2] Allen, H. \& Taylor, M. P. (1990). Charts, noise and fundamentals in the London foreign exchange market. The Economic Journal 100(400): 49-59.

[3] Angeletos, G-M \& Werning, I. (2006). Crises and prices: information aggregation, multiplicity, and volatility. The American Economic Review 96(5): 1720-1736.

[4] Baillie, R. T. (1996). Long memory processes and fractional integration in econometrics. Journal of Econometrics 73(1): 5-59.

[5] Bauer C., De Grauwe P. and Reitz S. (2009). Exchange rate dynamics in a target zone - A heterogeneous expectations approach. Journal of Economic Dynamics \& Control 33, 329344.

[6] Bollerslev, T., Osterrieder, D., Sizova, N. \& Tauchen, G. (2013). Risk and return: Long-run relations, fractional cointegration, and return predictability. Journal of Financial Economics 108(2): 409-424.

[7] Bouchaud, J.-P., A. Matacz, and M. Potters. 2001. Leverage effect in financial markets: The retarded volatility model. Physical Review Letters 87 (22): 228701.

[8] Brock, W. A. \& Hommes, C. H. (1998). Heterogeneous beliefs and routes to chaos in a simple asset pricing model. Journal of Economic Dynamics and Control 22(8-9): 1235-1274.

[9] Campbell, J. Y., Lo, A. W. \& MacKinlay, C. (1997). The Econometrics of Financial Markets. Princeton University Press.

[10] Chiarella, C., He, X., Huang, W. \& Zheng, H. (2012). Estimating behavioural heterogeneity under regime switching. Journal of Economic Behavior \& Organization 83(3): 446-460. 
[11] Christensen, B. J. \& Nielsen, M. ø. (2007). The Effect of Long Memory in Volatility on Stock Market Fluctuations. Review of Economics and Statistics 89(4): 684-700.

[12] Christensen, B. J., Nielsen, M. Nø. \& Zhu, J. (2010). Long memory in stock market volatility and the volatility-in-mean effect: The FIEGARCH-M Model. Journal of Empirical Finance 17(3): 460-470.

[13] Cont, R. (2001). Empirical properties of asset returns: stylized facts and statistical issues. Quantitative Finance 1 (2), 223-236.

[14] Day, R. H. \& Huang, W. (1990). Bulls, bears and market sheep. Journal of Economic Behavior \& Organization 14(3): 299-329.

[15] De Grauwe P., Dewachter H. and Embrechts M. (1993): Exchange Rate Theory - Chaotic Models of Foreign Exchange Markets. Blackwell, Oxford.

[16] de Jong, E., Verschoor, W. F. C. \& Zwinkels, R. C. J. (2010). Heterogeneity of agents and exchange rate dynamics: Evidence from the EMS. Journal of International Money and Finance 29(8): 1652-1669.

[17] Dieci R. and Westerhoff F. (2010): Heterogeneous speculators, endogenous fluctuations and interacting markets: a model of stock prices and exchange rates. Journal of Economic Dynamics \& Control, 34, 743-764.

[18] Ebrahim, M. \& Mathur, I. (2001). Investor Heterogeneity, Market Segmentation, Leverage, and the Equity Premium Puzzle. Journal of Banking and Finance 25(10): 1897-1919.

[19] Frankel, J. A. \& Froot, K. A. (1990). Chartists, Fundamentalists, and Trading in the Foreign Exchange Market. The American Economic Review 80(2): 181-185.

[20] Gabaix, X., Parameswaran G., Vasiliki P. \& H. Eugene S. (2003). A Theory of Power Law Distributions in Financial Market Fluctuations. Nature, 423: 267-70. 
[21] Gabaix, X., Parameswaran G., Vasiliki P. \& H. Eugene S. (2006). Institutional Investors and Stock Market Volatility. Quarterly Journal of Economics 121 (2): 461-504.

[22] Gaunersdorfer A. and Hommes C.H. (2007): A nonlinear structural model for volatility clustering. In: Teyssière G. and Kirman A. (eds.): Long Memory in Economics. Springer, Berlin, 265-288."

[23] Geweke, J. \& Porter-Hudak, S. (1983). The estimation and application of long memory time series models, Journal of Time Series Analysis 4(4): 221-238.

[24] He, X. \& Westerhoff, F. H. (2005). Commodity markets, price limiters and speculative price dynamics. Journal of Economic Dynamics and Control 29(9): 1577-1596.

[25] He, X. and Y. Li (2007), Power-law behaviour, heterogeneity, and trend chasing, Journal of Economic Dynamics and Control. 31, 3396-3426

[26] He, X. \& Y. Li (2015), Testing of a market fraction model and power-law behaviour in the DAX 30, Journal of Empirical Finance, 31, 1-17.

[27] Huang, W., Zheng, H. \& Chia, W.-M. (2010). Financial crises and interacting heterogeneous agents. Journal of Economic Dynamics and Control 34(6): 1105-1122.

[28] Huang, W., Zheng, H. \& Chia, W.-M. (2012). Asymmetric returns, gradual bubbles and sudden crashes. The European Journal of Finance 19(5): 420-437.

[29] Jegadeesh, N. and Titman, S. (1993), Returns to Buying Winners and Selling Losers: Implications for Stock Market Efficiency. The Journal of Finance, 48: 65-91.

[30] Jongen, R., Verschoor, W. F. C., Wolff, C. C. P. \& Zwinkels, R. C. J. (2012). Explaining dispersion in foreign exchange expectations: A heterogeneous agent approach. Journal of Economic Dynamics and Control 36(5): 719-735.

[31] Kasa, K., Walker, T. B. \& Whiteman, C. H. (2014). Heterogeneous Beliefs and Tests of Present Value Models. The Review of Economic Studies 81 (3): 1137-1163. 
[32] Lo, A. W. (1991). Long-Term Memory in Stock Market Prices. Econometrica 59(5): 12791313.

[33] Lof, M. (2012). Heterogeneity in stock prices: A STAR model with multivariate transition function. Journal of Economic Dynamics and Control 36(12): 1845-1854.

[34] Lof, M. (2015). Rational Speculators, Contrarians, and Excess Volatility. Management Science 61(8), 1889-1901 .

[35] Lux, T. (1995). Herd Behaviour, Bubbles and Crashes. The Economic Journal 105(431): $881-896$

[36] Moskowitz, T., Ooi, Y. H. \& Pedersen, L. H. (2012), Time series momentum. Journal of Financial Economics 104, 228-250.

[37] Pagan, A. 1996. The econometrics of financial markets. Journal of Empirical Finance 3(1): $15 \mathrm{~V} 102$.

[38] Parke, W. R. and G. A. Waters (2007). An evolutionary game theory explanation of ARCH effects. Journal of Economic Dynamics and Control 31(7): 2234-2262.

[39] Phillips, P. C. B. (2007). Unit root log periodogram regression. Journal of Econometrics 138(1): 104-124.

[40] Poterba, J. M. and L. H. Summers (1988). Mean reversion in stock prices. Journal of Financial Economics 22(1): 27-59.

[41] Robinson, P. M. (1995). Log-Periodogram Regression of Time Series with Long Range Dependence. The Annals of Statistics 23(3): 1048-1072.

[42] Rossi, E. \& Santucci de Magistris, P. (2013). Long memory and tail dependence in trading volume and volatility. Journal of Empirical Finance 22(0): 94-112. 
[43] Taylor, S. (2007). Asset price dynamics, volatility, and prediction. Princeton, N.J. : Princeton University Press.

[44] Tsay, R. S. (2010). Analysis of Financial Time Series, 3rd Edition. Wiley. 